NASA Technical Memorandum 109139

ARL Memorandum Report 157
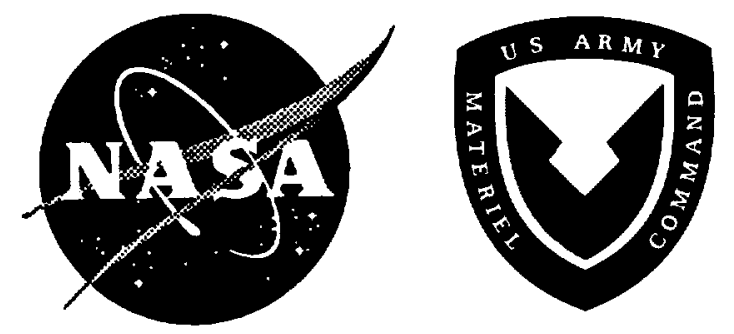

\title{
Optimization for Minimum Sensitivity to Uncertain Parameters
}

Jocelyn I, Pritchard

Vehicle Structures Directorate, ARL, Langley Research Center, Hampton, Virginia

Howard M. Adelman

Langley Research Center, Hampton, Virginia

Jaroslaw Sobieski

Langley Research Center, Hampton, Virginia

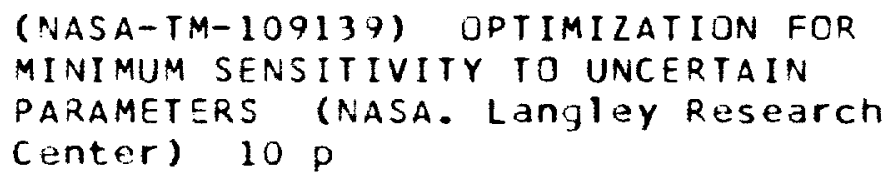

July 1994

National Aeronautics and Space Administration Langley Research Center Hampton, Virginia 23681-0001 
$\because \quad-$

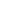




\title{
OPTIMIZATION FOR MINIMUM SENSITIVITY TO UNCERTAIN PARAMETERS
}

\author{
Jocelyn I. Pritchard ${ }^{*}$, Howard M. Adelman ${ }^{\dagger}$, and Jaroslaw Sobieszczanski-Sobieski $\ddagger$ \\ NASA Langley Research Center, Hampton, Virginia 23681
}

\begin{abstract}
A procedure to design a structure for minimum sensitivity to uncertainties in problem parameters is described. The approach is to directly minimize the sensitivity derivatives of the optimum design with respect to fixed design parameters using a nested optimization procedure. The procedure is demonstrated for the design of a bimetallic beam for minimum weight with insensitivity to uncertainties in structural properties. The beam is modeled with finite elements based on two dimensional beam analysis. A sequential quadratic programming procedure used as the optimizer supplies the Lagrange multipliers that are used to calculate the optimum sensitivity derivatives. The procedure is validated by comparing the optimization results to parametric studies.
\end{abstract}

\section{Introduction}

Parameters are characteristics of an optimization problem that are held constant during the optimization process in which the design variables are changing. In practical design situations the exact values of the parameters may not be known (e.g., loads, material properties, manufacturing errors, etc.). Uncertainties in parameters may cause variations in the outcome of the design process; for example, uncertainties in the constraints may cause the design to be unacceptable. Unfortunately, in many cases the uncertainties are very difficult and costly to control. It would be advantageous to approach the design lask using the key principle of quality design as formulated by Taguchi who proposed that designs be as insensitive as possible to variations in parameters that affect their behavior. ${ }^{1}$

Many efforts have been directed toward developing design processes wherein the performance of the final product will be minimally affected by variations in parameters. Several efforts have been made to approach the problem by imposing safety margins on the

\footnotetext{
* Research Engineer, Army Research Laboratory, VSD, Member AHS

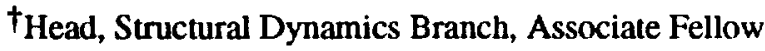
AIAA, Member AHS

$\ddagger_{\mathrm{MDO}}$ Coordinator, Structures Division, Fellow AIAA
}

constraints to avoid entering the infeasible region. For example, in reference 2 a worst case approach is used wherein a specified tolerance on the uncertain design variables reduces the feasible design space. The objective is to stay as close as possible to the original optimum and to remain within the newly defined feasible region. A padding factor is added to the constraints in reference 3 . The factor is proportional to the gradient of the constraint. The constraints are updated throughout the optimization process using linear and nonlinear approximations. In references 4 and 5 the authors proposed a method of designing for "feasibility robustmess". Given specified tolerances on design variables and parameters, variations in the constraints are predicted using worst case and statistical analysis. This maintains a certain probability of remaining feasible throughout the design process in spite of fluctuations in the variables and parameters.

The above methods effectively reduce the size of the feasible region resulting in trading a less optimum value of the objective function for a higher degree of robustness. In references 2,4 , and 5 this concept was enhanced by adding tolerance ranges on specified controllable variations as variables in the objective function so that the values of the tolerances were chosen during the design process. Reference 6 labeled this approach a "tolerance allocation problem". The method was applied to the design of a simple two-bar truss to obtain a trade-off between the tolerances on the structural dimensions and the manufacturing cost. As the tolerances decrease, the cost of manufacturing the product increases. In reference 7 a second order tolerance model was incorporated into a nonlinear optimization procedure which improved the formulation by including a measure of function skewness in the design process.

Another approach to decreasing the effects of uncertainties in design is to directly minimize the sensitivity of the design with respect to the uncertainties. Reference 8 minimized the sensitivity derivative of the response of a system with respect to uncertain parameters using shape design variables with formal optimization techniques. Reference 9 proposed that tradeoffs be made in the design objectives to ensure a degree of insensitivity to uncontrollable parameter variations. This approach was referred to as a "design for latitude" and it was handled with a multiple objective function minimization procedure which included the sensitivity of the design with respect to the uncertain parameters in the design objective. Reference 10 also used multiobjective optimization methods to minimize sensitivities to variations in design 
parameters by incorporating sensitivity derivatives of the objective and constraints into the objective function or by constraining them to be less than a specified value. Several formulations incorporating sensitivitybased constraints and/or sensitivity-based objectives were discussed. Reference 11 applied goal programming methods in a similar manner to minimize multiple objectives including the sensitivities of both fixed parameters and design variables to uncertainties. Reference 12 utilized fuzzy sets to define a multiple objective design optimization problem dealing with uncertainty.

The approach taken in this paper extends the concept of directly minimizing sensitivity derivatives to that of minimizing optimum sensitivity deriviatives $(\mathrm{OSD})^{13,14}$ with respect to problem parameters using a nested optimization procedure. The procedure is demonstrated for the design of a bimetallic beam for minimum weight with insensitivity to uncertain structural propeties. The beam is modeled with finite elements based on two dimensional beam analysis. An IMSL $^{15}$ sequential quadratic programming procedure used for the optimization supplied the Lagrange multipliers that were used to calculate the OSD using the method described in references 13 and 14.

\section{Approach}

The overall procedure contains two nested optimization loops as shown in Figure 1.

(1)

(2)

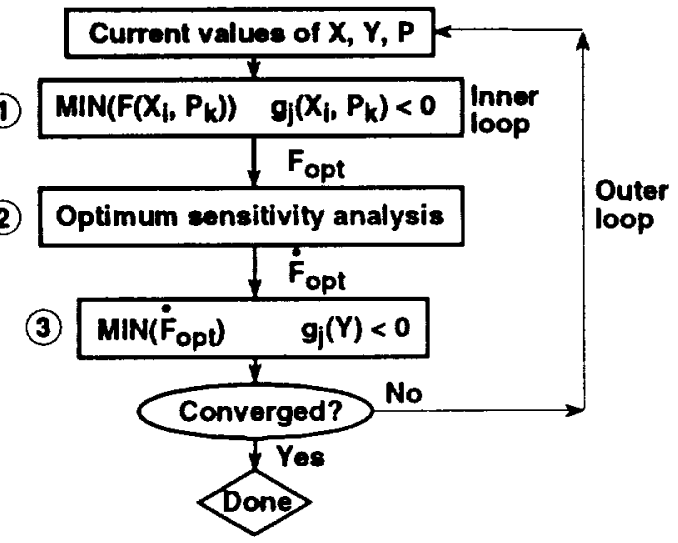

Fig. 1. Flowchart of overall procedure.

The uncertain inputs are represented by the constant parameters, $P$. The usual formulation of an optimization problem is solved within the inner loop (box 1) yielding a constrained optimum comprised of the optimal values of the objective function, $F$ and the design variables, $X$ denoted by $F_{\text {opt }}$ and $X_{O p t}$. These quantities are functions of the parameters $P$, therefore they are sensitive to uncertainties in these parameters. That sensitivity is quantified by the optimum sensitivity derivatives in box 2 . These derivatives are represented by $\mathrm{dF}_{\mathrm{opt}} / \mathrm{dP}$ or $\dot{\mathrm{F}}_{\mathrm{opt}}$. The derivatives may be computed by finite differencing on the optimization repeated with a parameter perturbed one at a time (expensive, inaccurate, but simple to implement) or by using a quasi-analytical method that is cheaper, more accurate, but requires more implementation effort. 13 The success of this procedure was found to be dependent on a highly converged inner loop optimization that provides accurate Lagrange multipliers. The outer loop minimizes the sensitivity of the outcome of the inner loop to the uncertain parameters by selecting the design associated with the smallest values for the optimum sensitivity derivatives (box 3 ). The objective function in the outer loop can be made up of multiple terms including OSD's with respect to several parameters and a term to reflect the value of the objective function in the inner loop. Weighting factors can be applied to each of these terms to prioritize their relative importance during the outer loop optimization. Such a weighted multiobjective function allows tradeoffs between the optimum design that is achieved in the inner loop and the level of sensitivity of this design to the uncertain parameters. A user may choose to accept a reduction in the measure of goodness (objective function) of the original design to gain a degree of robustness (insensitivity to uncertain parameters).

This optimization problem could be expensive to solve because there are two nested optimization loops. The computational expense could be amplified due to the fact that to minimize a sensitivity means to minimize a function that already depends on the derivatives. Use of a gradient-driven search algorithm would imply working with the computationally expensive second derivatives. Therefore, it was decided to use Powell's method ${ }^{16}$, a non-gradient-based search algorithm, in the outer loop.

\section{Example Problem}

A simple but cogent example is used to demonstrate the procedure and its potential. A beam of a fixed length $\mathrm{L}=100.0$ inches is clamped at both ends as shown in figure 2 .

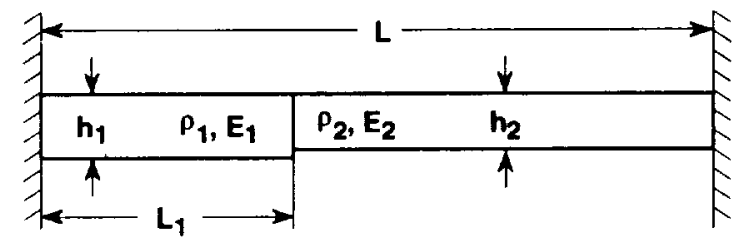

Fig. 2. Bi-material beam problem.

The beam is made up of two sections. From the left end to a distance $\mathrm{L}_{1}$ from the left end, the beam has Young's modulus $E_{1}$, a density $\rho_{1}$ and height $h_{1}$. The other section has $E_{2}, \rho_{2}$, and $h_{2}$. Both sections have rectangular cross-sections of width $B_{1}$ and $B_{2}$. The values of these material properties are given in table 1 . It is assumed that there is uncertainty in the values of 
$\rho_{1}$ and $E_{2}$. The objective is to design this beam using design variables $L_{1}, h_{1}$, and $h_{2}$ to have minimum

\begin{tabular}{|c|c|c|}
\hline \multicolumn{2}{|c|}{ Table 1 - Beam material properties } \\
\hline & Section 1 & Section 2 \\
\hline $\begin{array}{c}\text { Rho } \\
(\text { lbm/in }\end{array}$ & 0.100 & 0.056 \\
\hline $\begin{array}{c}\text { Youngs } \\
\text { modulus } \\
\text { (psi) }\end{array}$ & $2.0 \mathrm{E}+7$ & $1.0 \mathrm{E}+7$ \\
\hline $\begin{array}{c}\text { Width (in) } \\
\text { L }\end{array}$ & 4.0 & 4.0 \\
\hline \multicolumn{3}{|c|}{100 inches } \\
\hline
\end{tabular}

weight with constraints on the frequencies and to design it with minimum sensitivity to the uncertainty in the parameters, $\rho_{1}$ and $E_{2}$.

\section{Analytical model}

The beam was modeled with 10 finite elements which were based on a two dimensional beam analysis using bending elements. As the value of $\mathrm{L}_{1}$ was varied each section of the beam was divided into elements such that a joint always existed at $L_{1}$. Each section had a minimum of two elements.

\section{Inner Loop Formulation}

The inner optimization problem minimizes the weight of the beam subject to frequency constraints. The weight is given in equation 1 .

$$
F=\sum_{i=1}^{2} \rho_{i} L_{i} B_{i} h_{i}
$$

where $B_{i}, L_{j}$, and $h_{i}$ are the width, length, and height of the ith section of the beam, respectively. In the inner loop optimization, the design variables are the heights of the beam sections, $h_{1}$ and $h_{2}$, while $L_{1}$ is a constant parameter. Constraints place lower and upper bounds on the fundamental bending frequency, $\omega$ and are given in equation (2). Satisfied constraints are represented by the following expressions.

$$
1.0-\frac{\omega}{\omega_{1}} \leq 0 \text { and } \frac{\omega}{\omega_{u}}-1.0 \leq 0
$$

where $\omega_{1}$ and $\omega_{u}$ are the lower and upper bounds on the frequency.

An IMSL sequential quadratic programming (SQP) routine performs the optimization from which the Lagrange multipliers needed for subsequent calculation of the OSD's are output.

\section{Quter Loon Formulation}

In the outer loop the sensitivity of the inner loop optimum design is minimized with respect to the uncertainty in $\rho_{1}$ and $E_{2}$ using $L_{1}$ as the "auxiliary" design variable. In other words, of all the minimum weight designs that satisfy the constraints the objective is to find the one which is least sensitive to uncertainties in $\rho_{1}$ and $E_{2}$. Since there are two uncertain parameters, the sensitivity of the optimum weight design must be minimized with respect to both parameters. This can be done using a multiobjective function with the first two terms being the magnitudes of the two derivatives with a weighting factor $\beta$ as shown in equation 3.

$$
\begin{aligned}
\mathrm{OBJ}=\beta\left(\mathrm{DFOPT}_{1}\right) & +\beta\left(\mathrm{DFOPT}_{2}\right) \\
& +\delta\left|\frac{W-W^{*}}{W^{*}}\right|
\end{aligned}
$$

where DFOPT $_{1}$ and DFOPT $_{2}$ are normalized optimum sensitivity derivatives as shown in equation 4 .

$$
\begin{aligned}
& \text { DFOPT }_{1}=\frac{\rho_{1}}{F_{\text {opt }}} \frac{d F_{\text {opt }}}{d \rho_{1}} \\
& \text { DFOPT }_{2}=\frac{E_{2}}{F_{\text {opt }}} \frac{d F_{\text {opt }}}{d E_{2}}
\end{aligned}
$$

These normalized derivatives, sometimes referred to as logarithmic derivatives, are valuable in that they measure a percent change in the objective function due to a percent change in the design variables. The optimum sensitivity derivatives are calculated from equation 5 using the quasi-analytical method of references 13 and 14 .

$$
\frac{\mathrm{dF}_{\text {opt }}}{\mathrm{dP}}=\frac{\partial \overline{\mathrm{F}}}{\partial \mathrm{P}}+\bar{\lambda}^{\mathrm{T}} \frac{\partial \overline{\mathrm{g}}_{\mathrm{a}}}{\partial \mathrm{P}}
$$

The overbars are used to denote quantities at the optimum and $\overline{\mathrm{g}}_{\mathbf{a}}$ represents the constraints that are active at the optimum, $\lambda$ is the Lagrange multiplier, and $P$ represents the parameter.

The last term in equation 3 represents the tradeoff between a low weight design and the insensitivity to the uncertain parameters. The term $W^{*}$ is a reference weight and $\delta$ is a weighting factor. The values of $\delta$ and $\beta$ which are problem dependent are chosen arbitrarily. A rational method for obtaining the value of $\mathrm{W}^{*}$ is by optimizing the weight of the beam subject to frequency constraints using inner and outer loop design variables simultaneously. The value of $W^{*}$ will have an effect on the location of the optimum and the nature of the curve in the vicinity of the optimum. 
The objective function $O B J$ is minimized using the auxiliary design variable $L_{1}$. Minimization of the optimum sensitivity is done using Powell's Method which for one design variable degenerates into a onedimensional search.

\section{Results}

\section{Results of Optimization Procedure}

In order to assess the validity of the nested optimization procedure, a study was conducted in which the parameter $\mathrm{L}_{1}$ was changed in discrete increments in the outer loop and only the inner loop optimization was performed. A curve that represents a series of discrete calculations of OBJ versus . 5 inch increments of $\mathrm{L}_{1} / \mathrm{L}$ is shown in figure 3 for $\beta$ equal to $10, W^{*}$ equal to 30 and $\delta$ equal to 1 . Each data point represents a minimum weight

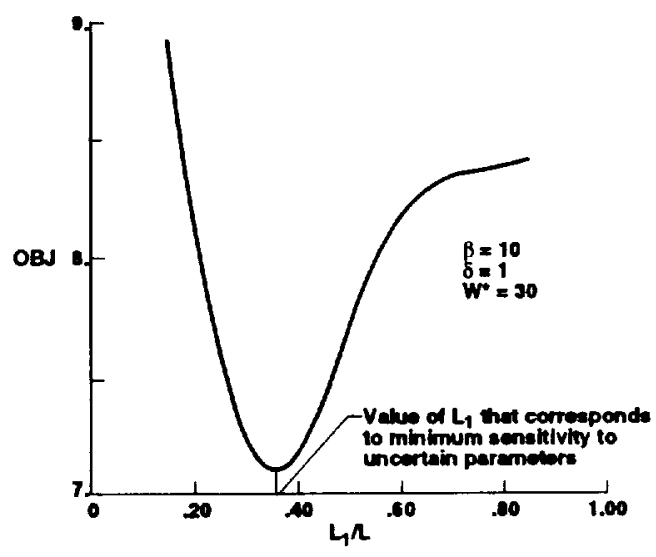

Fig. 3. Curve of discrete calculations of outer loop objective function OBJ versus $\mathbf{L}_{\mathbf{1}} / \mathbf{L}$.

optimization performed in the inner loop and an optimum sensitivity analysis calculation. The minimum indicated in the figure corresponds to the value of $L_{1} / \mathrm{L}$ for which the beam has a minimum weight design and is least sensitive to uncertainties in the parameters $\rho_{1}$ and $E_{2}$. From the graph, the data point at minimum $O B J$ is $L_{1} / L$ is .365 . The nested optimization procedure produced a value for $L_{1} / L$ equal to .364 . Values associated with the initial and final designs are given in table 2 . The values in the table

\begin{tabular}{|c|c|c|c|c|c|c|c|}
\hline$w=30$ & $L_{1}$ & $h_{1}$ & $h_{2}$ & Woight & DFOPT, $_{1}$ & DFOPT $_{2}$ & OBJ \\
\hline Initial & 50.0 & 0.533 & 3.544 & 50.800 & .379 & 321 & 7.695 \\
\hline Final & 36.43 & 0.283 & 4.654 & 70.000 & .132 & .42 & 7.000 \\
\hline
\end{tabular}

indicate that by adding approximately 40 percent more weight to the beam, the sensitivity derivative of the optimum weight with respect to $\rho_{1}$ (DFOPT 1 ) was decreased by nearly a factor of 3 . The optimum sensitivity derivative with respect to $\mathrm{E}_{2}\left(\mathrm{DFOPT}_{2}\right)$ was increased slightly. This trend may be altered depending upon the choice of the weighting factors $\beta$ and $\delta$. In this case $\beta$ was considerably large and weighted both optimal sensitivity derivatives equally in the outer loop objective function whereas $\delta$ was comparatively small. It may be advantageous to use a different weighting factor for each optimum sensitivity derivative to emphasize minimization of one or the other or to increase the value of $\delta$ to emphasize minimization of the weight. The following section will discuss the role of the weighting factors in tailoring the formulation of the problem to alter the optimum design.

\section{Tradeoffs Using Weighting Factors}

The weighting factors play a crucial role in the outcome of the process. Figure 4 shows four graphs, the first three representing one of the three individual terms in the outer loop objective function versus $L_{1} / \mathrm{L}$ and the fourth showing the optimum values of the beam heights versus $L_{1} / L$. The figure clearly shows that in

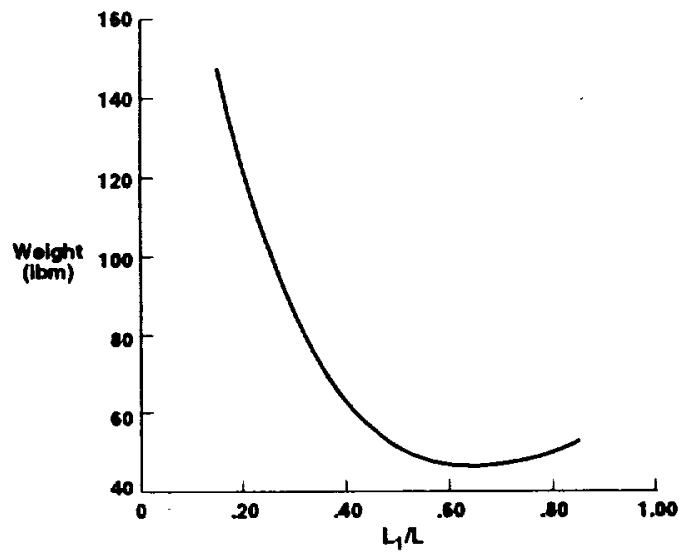

(a) Weight from inner loop optimization versus $L_{1} / L$

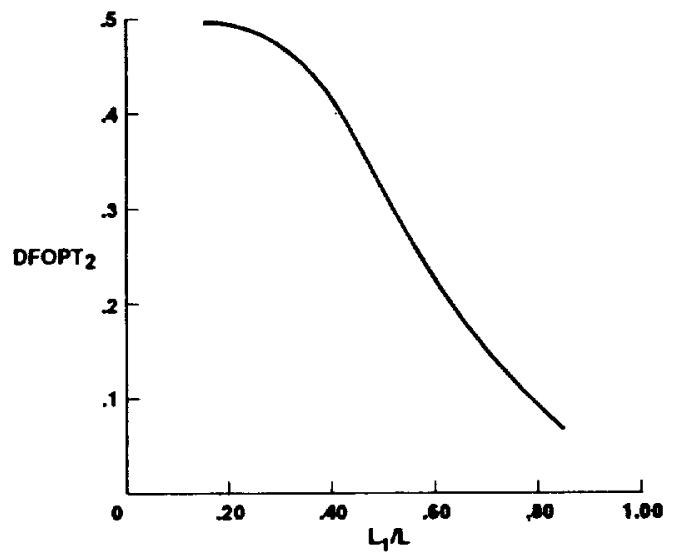

(b) Optimum sensitivity calculated at inner loop optimum versus $L_{1} / L$ 


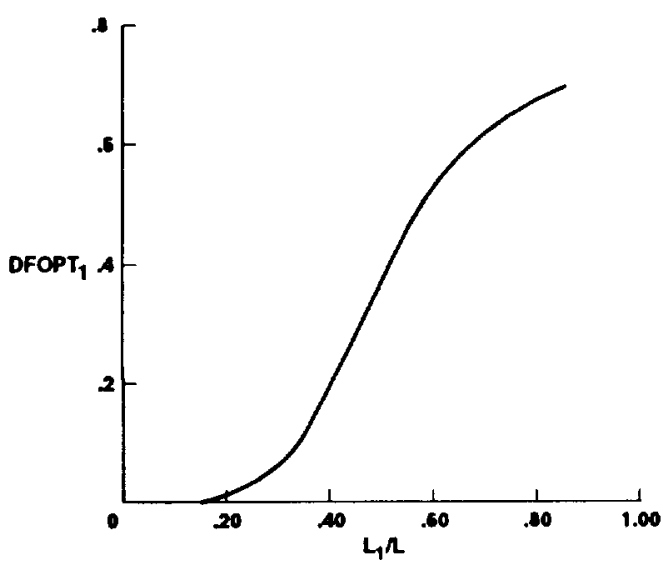

(c) Optimum sensitivity calculated at inner loop optimum versus $\mathrm{L}_{1} / \mathrm{L}$

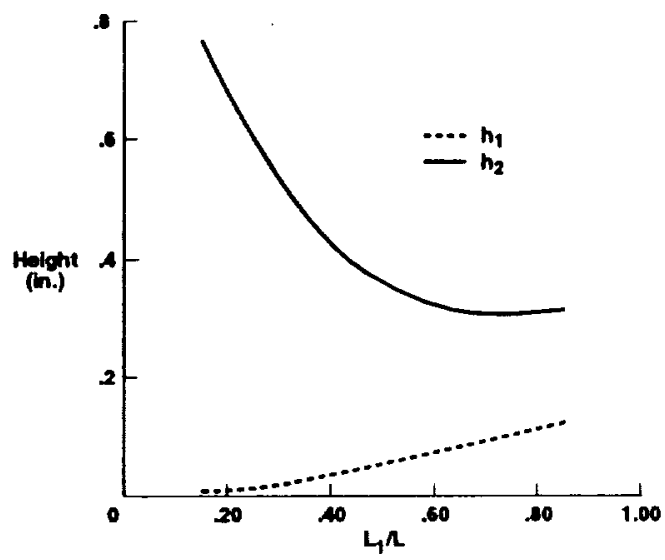

(d) Height of beam sections from inner loop optimizations versus $L_{1} / L$

Fig. 4. Graphs of the three components of the outer loop objective function and the heights of the beam sections versus $L_{1} / L$.

order to minimize each individual term, contradicting values of $L_{1} / L$ are needed. For instance, in figure $4 a$ which shows the optimum weight versus $L_{1} / \mathrm{L}$ demands that $\mathrm{L}_{1} / \mathrm{L}$ be about 64 percent. Figure $4 \mathrm{~b}$ indicates that the optimum sensitivity derivative with respect to $\mathrm{E}_{2}$ (DFOPT 2 ) wants to push $\mathrm{L}_{1} / \mathrm{L}$ all the way to the right end of the beam. On the other hand, figure $4 c$ shows that in order to decrease the optimum sensitivity derivative with respect to $\rho_{1}$ (DFOPT 1 ), $\mathrm{L}_{1} / \mathrm{L}$ should be located near the left end of the beam. Obviously a value for $L_{1} / L$ has to be chosen that represents a compromise in minimizing all three terms simultaneously.

The following example demonstrates the effect of the weighting factors on the final design. Similar to the first example, a series of discrete calculations of the outer loop composite objective function $\mathrm{OBJ}$ versus 0.5 inch increments of $L_{1} / L$ were carried out for two cases. In the first case $\beta=5$ and $\delta=1$ for $W^{*}$ equal to 30 . In the second case $\beta=1$ and $\delta=5$ for $W^{*}=30$. The plots are shown in figure 5 . Also shown in figure 5 is a curve representing the optimum weight (normalized with respect to $\mathrm{W}^{*}$ ) from the inner loop for each incremental value of $L_{1} / \mathrm{L}$. Investigation of the figure shows that

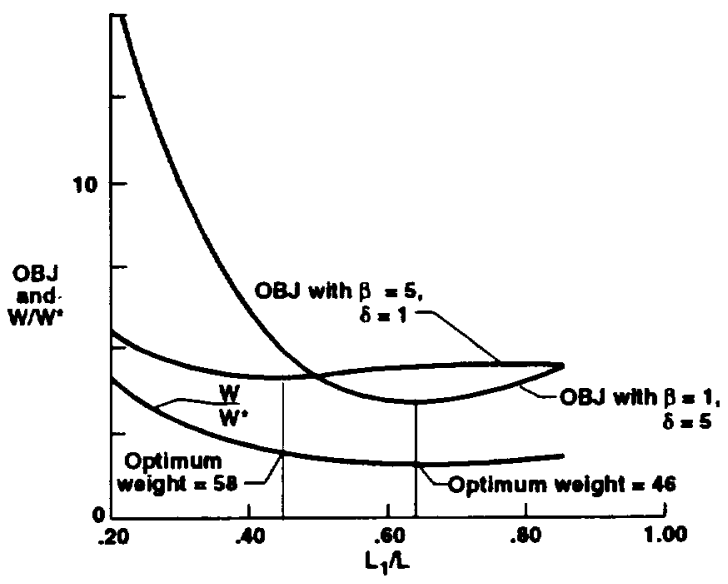

Fig. 5. Graph showing tradeoff of insensitivity versus weight.

the minima of the two $\mathrm{OBJ}$ curves correspond to different values of $L 1 / L$ and therefore different values of weight depending upon what values of the weighting factors are used. This is evident from figure 5 when comparing the weight values that correspond to the minima of the two curves. A large value of $\beta$ allows the procedure to add weight to the structure if it will reduce the sensitivity of the beam to the uncertain parameters. On the other hand if the sensitivity to the problem parameters is considered to be less important, the value of $\beta$ may be decreased and $\delta$ increased. In this case the value of $L_{1} / L$ that gives the minimum value of OBJ will correspond to a lower weight.

The optimization procedure was applied to this example problem for both cases involving values of $\delta$ and $\beta$ for $W^{*}=30 \mathrm{lbs}$. The procedure successfully located the values of $L_{1} / L$ that correspond to the minima shown in figure 5 for each case. Table 3 lists the initial and final values of $L_{1} / \mathrm{L}$, the heights of the sections, the weights of the beam, the values of the composite outer loop objective function (OBJ), and the optimal sensitivity derivatives for both cases. In the

\begin{tabular}{|c|c|c|c|c|c|c|c|}
\hline$W^{*}=30$ & $L_{1}$ & $h_{1}$ & $h_{2}$ & Weight & DFOPT $_{1}$ & DFOPT $_{2}$ & OBJ \\
\hline $\begin{array}{l}\text { Inlitid } \\
\beta=5 \\
\delta=1 \\
w^{\circ}=30\end{array}$ & 50 & 533 & 3.584 & 50.000 & .970 & .321 & 4.125 \\
\hline $\begin{array}{l}\text { Final } \\
0=5 \\
\delta=1 \\
W^{*}=30\end{array}$ & 43 & .403 & 4.027 & 50.341 & .247 & 318 & 4.119 \\
\hline $\begin{array}{l}\text { Initial } \\
\beta=1 \\
\delta=5 \\
W^{*}=30\end{array}$ & 50 & 593 & 3.584 & 50.000 & .370 & .321 & 4.160 \\
\hline $\begin{array}{l}\text { Final } \\
p=1 \\
\delta=5 \\
W^{+}=30\end{array}$ & 64 & .205 & 4.154 & 46.053 & 577 & .186 & 3,440 \\
\hline
\end{tabular}


case where $\beta$ was larger than $\delta$, a 15 percent increase in the weight occurred for a 34 percent decrease in $\mathrm{DFOPT}_{1}$ and a 20 percent increase in DFOPT 2 . For the second case where $\delta$ was larger than $\beta$, a 9 percent decrease in the weight was achieved for a 52 percent increase in DFOPT 1 and a 38 percent reduction in $\mathrm{DFOPT}_{2}$. In this example the individual optimal sensitivity derivatives had opposite trends. As one was decreased the other was increased. By assigning different weighting factors to the optimum sensitivity terms, one sensitivity or the other may be emphasized for reduction. The OBJ curve for case 1 was a relatively flat curve and therefore the reduction in the value of $\mathrm{OBJ}$ for case $\mathbf{1}$ was small compared to that of case 2 for any choice of the initial $L_{1} / L$ value.

\section{Concluding Remarks}

A procedure based on minimizing a linear combination of optimum sensitivity derivatives has been developed and tested on a simple beam problem. The results show that the procedure has the capability to successfully perform structural optimization which also minimizes the effects of uncertain parameters. It was important in the procedure to include a term that provided a measure of the minimum weight optimization along with optimum sensitivity terms in the overall objective function to gain control over the trade-off between a low-weight design and the insensitivity to uncertainties. The procedure provides insight into design tradeoffs among various uncertainties as well as tradeoffs between insensitivity and structural weight. The design space was studied and local minima were found to exist depending upon the starting values of the inner loop design variables for each consecutive inner loop optimization.

\section{Acknowledgment}

The authors wish to express their special thanks to Professor R. T. Haftka of Virginia Polytechnic Institute and State University whose contributions were essential during the course of this research.

\section{Appendix: Examination of Local Minima in the Design Space}

For the results shown in figures 3-5, the inner loop optimization is always initiated using the final values of the design variables from the previous inner loop optimization. This procedure produced smooth curves as shown in the figures. However, during the course of

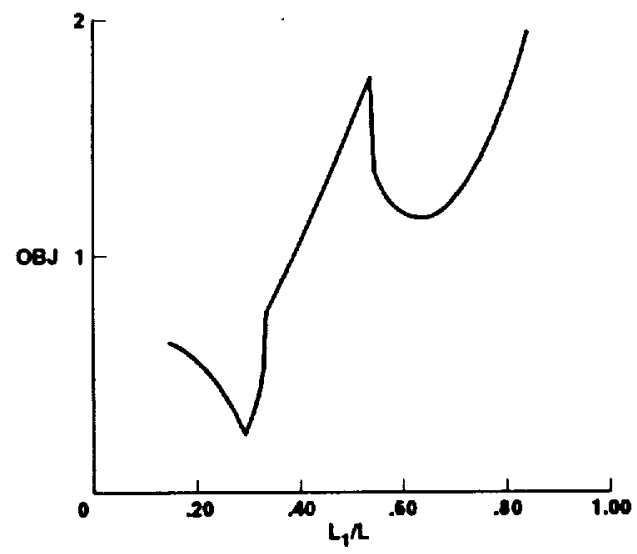

Fig. 6. Plot of outer loop objective function versus $L_{1} / L$ showing discontinuities in function.

this study the inner loop optimizations were also initiated using some fixed nominal values for the heights of the beam sections. The plots of the outer loop objective function versus $\mathrm{L}_{1} / \mathrm{L}$ exhibited discontinuities as shown for a typical case in figure 6. These discontinuities suggested that local minima were present in this example problem. While investigating

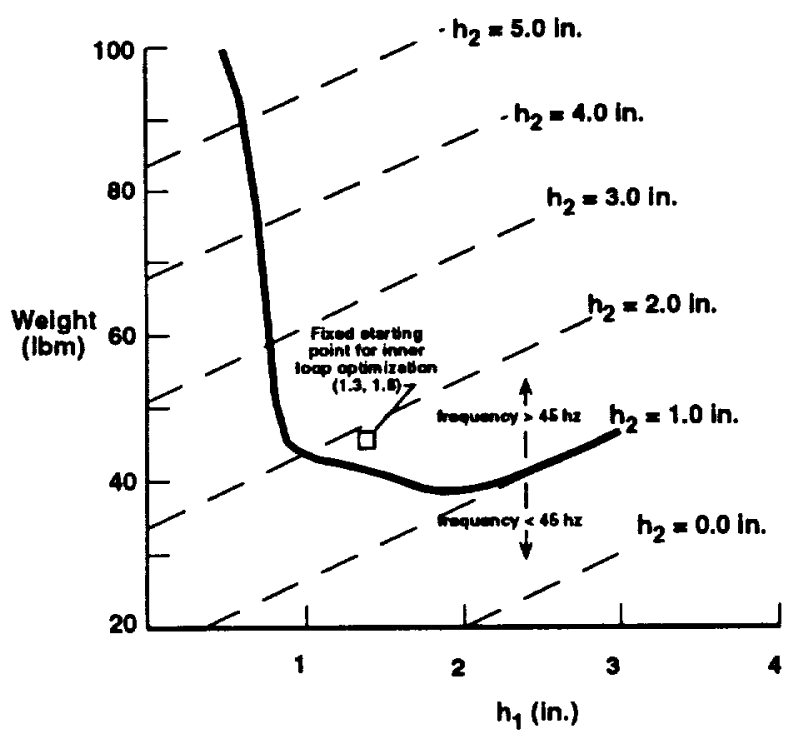

(a) $\mathrm{L}_{1} / \mathrm{L}=.25$ 


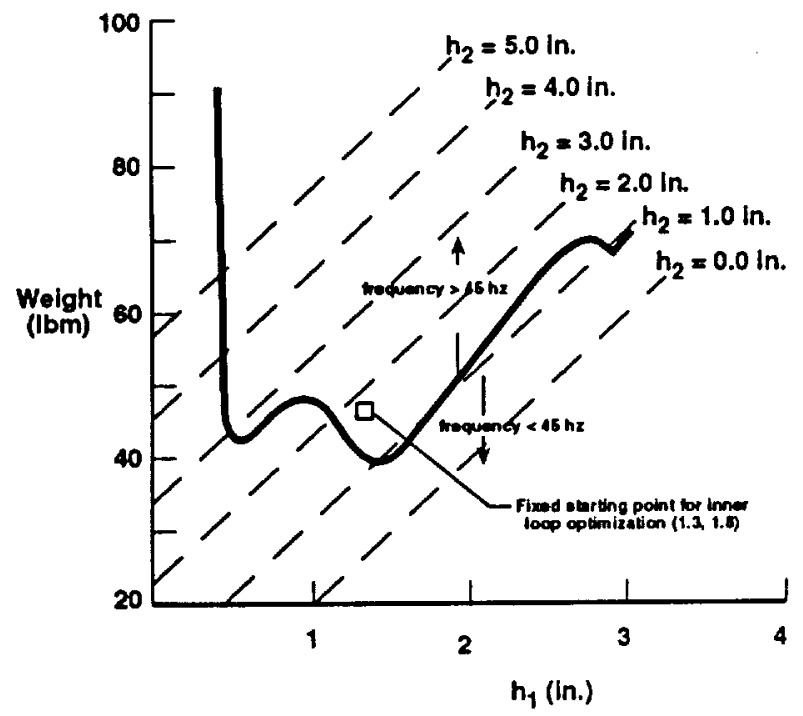

$\mathrm{L}_{1} / \mathrm{L}=.50$

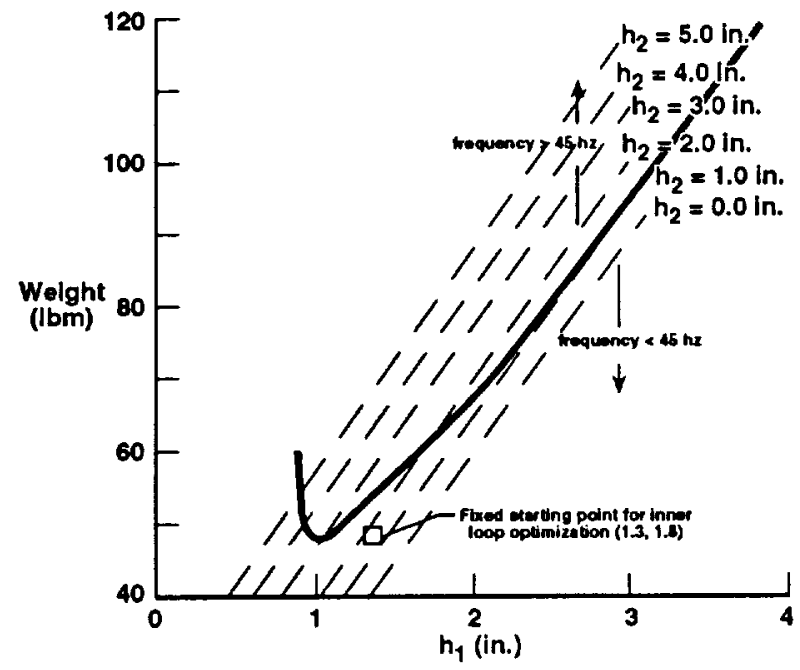

(c) $\mathrm{L}_{1} / \mathrm{L}=.75$

Fig. 7. Weight versus beam segment heights at lower bound constraint on frequency for $\mathrm{L}_{1} / \mathrm{L}=.25, .50$, and .75 .

this local minima phenomenon, it was observed that at the inner loop optimum the lower bound frequency constraint was active for all values of $L_{1}$. This constraint boundary is shown in figure 7 where the weight is plotted versus beam segment heights for three values of $L_{1} / L$. In figure $7 a$ and $7 c$ which represent values of $L_{1} / \mathrm{L}$ near the left and right ends of the beam respectively there is one minimum. Figure $7 \mathrm{~b}$ shows that for values of $L_{1} / L$ in the vicinity of the beam center as many as three local minima may exist. As $\mathrm{L}_{1} / \mathrm{L}$ changes the inner loop optimization may converge to a different local optimum depending upon the initial values of the design variables hence discontinuities will appear in the outer loop objective function. If the inner loop is initiated with design variables that are far away from the most current inner loop optimum the optimization algorithm can cause the procedure to find different local minima as $\mathrm{L}_{1} / \mathrm{L}$ is changed. Starting successive inner loop optimizations close to the optimum of the previous pass through the inner loop lead to convergence to a local minimum.

It should be noted that the discontinuities in the sweeps of the outer loop objective function versus $L_{1}$ shown in figure 6 did not cause the procedure to fail because the search algorithm in the outer loop did not require the calculation of any derivatives. However, the potential did exist for the overall procedure to converge to a local minimum which was not a global minimum.

\section{References}

1. Byrne, D. M., and Taguchi, S., " The Taguchi Approach To Parameter Design," Quality Progress, pp. 19-26, Dec. 1987.

2. Balling, R. J., Free, J. C., Parkinson, A. R., "Consideration Of Worst-Case Manufacturing Tolerances In Design Optimization," ASME Journal of Mechanisms, Transmission and Automation in Design, Vol. 108, No. 4, pp. 438-441, Dec. 1986.

3. Bennett, J. A. and Lust, R. V., "Conservative Methods For Structural Optimization," AIAA Joumal, Vol. 28, No. 8, pp. 1491-1496, Aug. 1990.

4. Parkinson, A., Sorensen, C., Free, J., Canfield, B., "Tolerances And Robustness In Engineering Design Optimization," Proceedings of the 16th ASME Design Automation Conference, Chicago, Ill., Sept. 1990, DEVol. 23-1.

5. Parkinson, A., Pourhassan, N., Sorensen, C., Tolerances and Robustness in Engineering Design Optimization: Further Results," Proceedings of the Design Productivity International Conference, Feb. 1991.

6. Teng, A., Free, J., Parkinson, A., "An Approach To Robust Design Of Dynamic Systems-Allocation Of Variation, "Proceedings of the 4 th AIAAJUSAF/NASA/OAI Symposium on Multidisciplinary Analysis and Optimization, Technical Papers, Part 2, Cleveland, OH., pp. 810-819, Sept. 1992.

7. Lewis, L., Parkinson, A., "Robust Optimal Design Using a Second Order Tolerance Model," Research in Engineering Design, Oct. 1993.

8. Belegundu, A. D., and Zhang, S., "Robust Mechanical Design Through Minimum Sensitivity," Proceedings of 15th ASME Design Automation Conference, Montreal, Canada, pp. 233-239, Sept. 1989. 
9. Sandgren, E., Gim, G., Ragsdell, K.M., "Optimal Design of a Class of Welded Structures Based on

Design for Latitude," ASME Journal of Mehanisms, Transmissions and Auromation in Design, Vol. 107, pp. 482-487, Dec. 1985.

10. Rao,S. S., Sundararaju, K., Balakrishna, C., Prakash, B. G., "Multiobjective Insensitive Design Of Structures," Computers and Strcutures, Vol. 45, No. 2., pp.349-359, 1992

11. Sandgren, E., "A Multi-Objective Tree Approach For Optimization Under Uncertainy," presented at the 15th Design Automation Conference, Montreal,

Canada, Sept., 1989, DE-Vol. 19-2.

12. Mohandas, S. U., Sandgren, S., "Multiobjective Optimization Dealing With Uncertainty," presented at the 15th Design Automation Conference, Montreal, Canada, Sept., 1989, DE-Vol. 19-2.

13. Sobieszczanski-Sobieski, J., Barthelemy, J. F., "Sensitivity fo Optimum Solutions to Problem Parameters," AIAA Journal, Vol. 20, No. 9, pp. 12911299, Sept. 1982.

14. Barthelemy, J. F., Sobieszczanski-Sobieski, J. "Optimum Sensitivity Derivatives of Objective Functions in Nonlinear Programming," AIAA Journal, Vol. 21,No. 6, pp. 913-915, June 1983.

15. International Mathematical and Statistical Library, IMSL, Houston, Texas, 1989.

16. Fox, R. L., Optimization Methods For Engineering Design, Addison-Wesley, Reading, MA., June 1973 



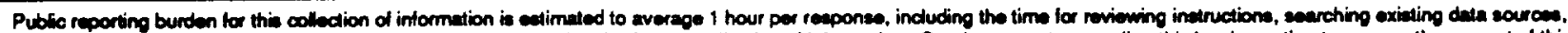

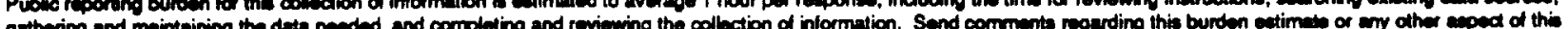
patherion

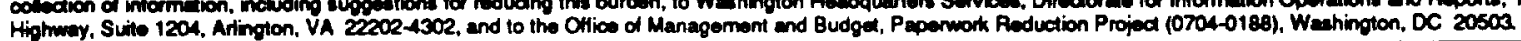

\begin{tabular}{|l|l|l}
\hline 1. AGENCY USE ONLY (Lanve blank) & $\begin{array}{l}\text { 2. REPORT DATE } \\
\text { July } 1994\end{array}$ & $\begin{array}{c}\text { 3. REPORT TYPE AND DATES COV } \\
\text { Technical Memorandum }\end{array}$
\end{tabular}

\section{TITLE AND SUBTITE}

Optimization for Minimum Sensitivity to Uncertain Parameters

\section{FUNDING NUMEERS}

505-63-36-06

\section{AUTHOR(S) \\ Jocelyn Pritchard" \\ Howard M. Adelman \\ Jaroslaw Sobieski}

7. PERFORMING ORGANIZATION NAME(S) AND ADDRESS(ES)

Army Research Laboratory, Vehicle Structures Directorate

Langley Research Center 23681-0001

NASA Langley Research Center

Hampton, VA 23681-0001

9. SPONSORING / MONTORING AGENCY NAME(S) AND ADDRESS(ES)

National Aeronautics and Space Administration

Washington DC 20546-0001

\section{U. S. Army Research Laboratory \\ Adelphi, MD 20783-1145}

11. SUPPLEMENTARY NOTES

Will be presented at the 5th AIAAUSAF/NASAISSMO Symposium on Multidisciplinary Analysis and Optimization, Panama City, FL, September 7-9, 1994 ARL-VSD

12a. DISTRIBUTION I AVAILABILTTY STATEMENT
Unclassified - Unlimited
Subject Category 05

\section{ABSTRACT (Maximum 200 words)}

The paper describes a procedure to design a structure for minimum sensitivity to uncertainties in problem parameters. The approach is to directly minimize the sensitivity derivatives of the optimum design with respect to fixed design parameters using a nested optimization procedure. The procedure is demonstrated for the design of a bimetallic beam for minimum weight with insensitivity to uncertainties in structural properties. The beam is modeled with finite elements based on two dimensional beam analysis. A sequential quadratic programming procedure used as the optimizer supplies the Lagrange multipliers that are used to calculate the optimum sensitivity derivatives. The method was perceived to be successful from comparisons of the optimization results with parametric studies.

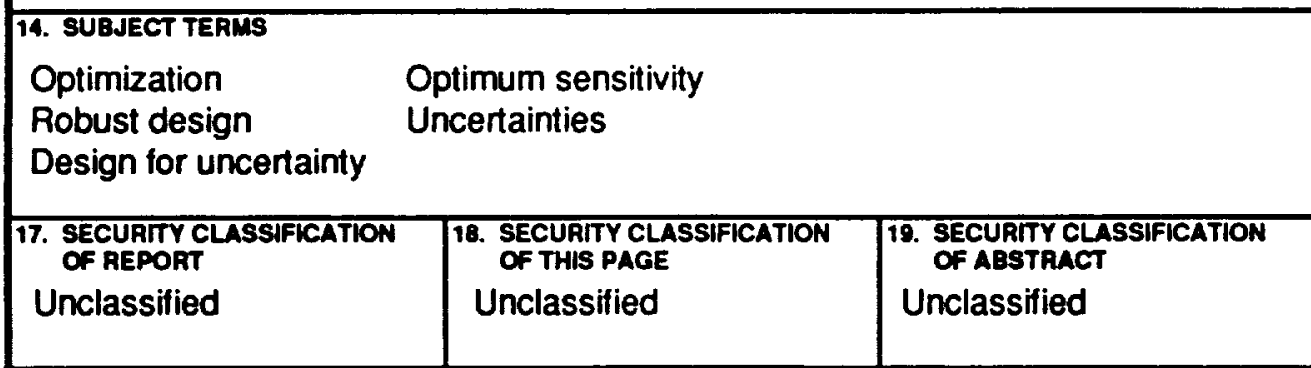

